\title{
Tradução, adaptação transcultural e validação da Escala de Exposição às Humanidades em estudantes de medicina
}

\section{Translation, transcultural adaptation and validation of a scale of exposure to humanities among medical students}

\author{
Ilse Seubert Coelho Vieira' ${ }^{10}$ | ilse.vieira@yahoo.com.br \\ Nathália Irffi Carvalho' (1) nathaliairffigmail.com \\ Antonio Carlos de Castro Toledo Júnior ${ }^{1}$ (D) toledoac@task.com.br \\ Eliane Perlatto Moura' (D) elianeperlatto@gmail.com
}

\section{RESUMO}

Introdução: As humanidades estão associadas à melhora de qualidades pessoais dos estudantes de Medicina. Não existem, até o momento, instrumentos de investigação que quantifiquem a exposição dos estudantes de Medicina às humanidades, e a disponibilidade de questionário com essas características, em português do Brasil, permite planejar e implementar estratégias e políticas educacionais que abordem esse tema.

Objetivo: Este estudo teve como objetivos realizar a tradução e adaptação transcultural do Humanities Score (Life Experiences + Attitudes), determinar sua validade e confiabilidade, e identificar o perfil de exposição às humanidades da população estudada.

Método: A versão original do questionário, em inglês, foi traduzida conforme preconizado na literatura, com adição ao instrumento de algumas atividades no campo das humanidades, por sugestão dos autores do instrumento original. O instrumento traduzido foi submetido ao pré-teste, com 31 estudantes de Medicina, para a validação semântica, e, a seguir, a versão final foi aplicada a 258 discentes. A análise fatorial exploratória foi aplicada para avaliar o instrumento, e utilizou-se o alfa de Cronbach para avaliar a consistência interna.

Resultados: O questionário final foi aplicado aos alunos para verificação do seu perfil de exposição às humanidades. Realizou-se análise estatística, e a versão final do instrumento denominado Escala de Exposição às Humanidades (EEH) constituiu-se de 17 itens com respostas do tipo Likert com cinco opções, cujo coeficiente alfa de Cronbach foi 0,689. A média de escore de exposição dos estudantes foi de 1,72 $\pm 0,37$, sendo influenciada por período em curso, realização de atividades sociais voluntárias, participação em grupos religiosos, prática de meditação e participação em atividades políticas. Observou-se ainda que as variáveis sexo feminino, exercício de atividades relacionadas com humanidades antes do curso e participar de cultos religiosos influenciaram positivamente na opinião sobre a importância atribuída às humanidades no currículo médico.

Conclusão: A EEH demonstrou estrutura e conteúdo confiáveis, permitindo correlacionar o comportamento dos alunos quanto à exposição às humanidades e a importância que atribuem à sua presença no currículo. Trata-se do primeiro instrumento que busca mensurar o índice de exposição às humanidades no Brasil, sendo necessários, entretanto, estudos adicionais para aprimorar a validação do instrumento.

Palavras-chave: Ciências Humanas; Estudantes de Medicina; Tradução; Estudo de Validação; Educação Médica.

\begin{abstract}
Introduction: The humanities are associated with the improvement of medical students' personal qualities. To date, there are no research instruments that quantify the exposure of medical students have to the humanities. Hence, the availability of a questionnaire with such characteristics in Brazilian Portuguese sets a precedent for the planning and implementation of educational strategies and policies addressing this topic.

Objective: to translate and transculturally adapt the "HUMANITIES SCORE (LIFE EXPERIENCES + ATTITUDES) questionnaire, determine its validity and reliability, as well as identify the type of exposure to the humanities of the assessed population.

Method: The original version of the questionnaire, written in English, was translated according to what is recommended by the specialized literature, with the addition of some activities in the humanities field, as per the original authors' suggestion. The translated instrument underwent a pre-test with 31 medical students for semantic validation, followed by the application of its final version to 258 students. The exploratory and the confirmatory factorial analyses were applied to assess the instrument with its internal consistency was checked with Cronbach's Alpha coefficient.

Result: The final questionnaire was administered to the students to verify their type of exposure to the humanities. After the statistical tests were carried out, the final version of the instrument, named "Escala de Exposição às Humanidades" (EEH), included 17 items with Likert-scale responses with five options each, and obtained a Cronbach's alpha of 0.689. The mean score of the students' exposure was $1.72 \pm 0.37$, being influenced by the period at medical school, the number of volunteer social activity experiences, the participation in religious groups, the practice of meditation, and the involvement in political activities. Variables such as female gender, engagement in previous actions related to the humanities before starting medical school and in religious ceremonies positively influenced the students' opinion about the importance of the humanities for the medical curriculum.
\end{abstract}

Conclusion: The EEH demonstrated reliability in its structure and content, allowing correlations between the students' exposure to humanities and their opinion about the importance of human sciences in the medical curriculum. It constitutes the first instrument that aims at measuring the humanities exposure rate in Brazil; however, further studies must be carried out, to better validate the instrument.

Keywords: Humanities; Medical Students; Translating; Validation Study; Medical Education.

1 Universidade José do Rosário Vellano, Belo Horizonte, Minas Gerais, Brasil.

Editora-chefe: Rosiane Viana Zuza Diniz. Editor associado: Maurício Abreu Pinto Peixoto.

Recebido em 02/02/21; Aceito em 15/10/21. Avaliado pelo processo de double blind review. 


\section{INTRODUÇÃO}

A relação entre a ciência e as humanidades existe ao longo do tempo e permeia o ensino e o aprendizado da medicina desde suas origens ${ }^{1}$. A concepção de "humanidades" remonta à Antiguidade clássica quando a educação estimulava a expansão da consciência do ser humano, no entanto a educação alcançava apenas uma parcela da população². Ao longo do tempo, o processo educativo estendeu-se a uma porção cada vez mais ampla da população, seguindo duas linhas básicas de aprendizado: a ciência, por um lado, e, por outro, as humanidades, estas constituindo a base insubstituível do próprio pensamento ${ }^{3}$. O termo humanismo surgiu na Renascença, e desde então os estudos tradicionais foram se tornando mais dinâmicos, baseados no programa studia humanitas: o homem renascentista foi em busca do conhecimento enciclopédico, para o desenvolvimento tanto do corpo quanto do espírito².

A regulamentação do ensino e da prática da medicina iniciou-se por meio de leis e decretos publicados pelo imperador Frederico II, na Itália de 1224. O currículo médico de então era composto de três anos de filosofia, cinco anos de medicina e um ano de prática².

Sendo a medicina frequentemente denominada "arte médica", seu aprendizado envolve, além do conhecimento técnico, aspectos diversificados, como comunicação, compreensão de fatores sociais, políticos, emocionais e espirituais que afetam a experiência humana, além de requerer a compreensão não só do adoecimento, mas também e essencialmente do sofrimento humano. Sir William Osler (1849-1919) defendeu intransigentemente o exercício de uma medicina ao mesmo tempo técnica e humana, e mesclou, com perfeição, a arte com a ciência médica ${ }^{4}$. E mesmo Abraham Flexner (1866-1959), em seu conhecido relatório que embasou a criação das escolas médicas modernas com foco no conhecimento biomédico e treinamento clínico, reconheceu a necessidade de uma reconfiguração da educação médica, recomendando uma resposta às mudanças nas circunstâncias científicas, sociais e econômicas, para o florescimento da formação médica de uma geração para a seguinte. A flexibilidade e a liberdade de mudar, de fato, faziam parte essencial de sua mensagem ${ }^{5,6}$.

As humanidades que se associam ao ensino da Medicina recebem diversas definições e delimitações, desde disciplinas curriculares comuns à formação em ciências humanas até a exposição às artes (exposição ativa, quando o indivíduo executa as atividades, ou passiva, quando ele as aprecia ${ }^{7}$ ). As artes relacionam-se, desde os primórdios, ao desenvolvimento humano e aumentam a empatia e as habilidades de comunicação, predispondo a posicionamentos éticos e ganhos em autoconceito e motivação, favorecendo níveis mais elevados de tolerância em relação ao outro ${ }^{8}$.

O estudante de Medicina necessita apreender um grande volume de habilidades instrumentais e emocionais, além de extensos conteúdos de cunho técnico-científico, ministrados em tempo integral. Daí ser escassa a disponibilidade de tempo para dedicar-se a atividades sociais, de lazer, esportivas e culturais, entre outras, o que interfere na qualidade de vida desses alunos ${ }^{9,10}$. Existem propostas para atenuação desse cenário, entre as quais alterações curriculares ${ }^{11,12}$, como a inserção de atividades correlatas às humanidades ${ }^{7,13}$. Ousager et al. $^{14}$ pontuam que há escolas médicas que incluem humanidades em seus currículos, mas que é difícil analisar, com algum grau de certeza, o quanto essa inserção efetivamente forma melhores médicos. Outros autores afirmam que os resultados dessas medidas atenuadoras carecem de uma análise mais objetiva ${ }^{15}$ e que a ausência de instrumentos hábeis para avaliar a efetividade das humanidades como estratégia educacional dificulta sua comprovação ${ }^{16}$.

É patente a importância do assunto, e constatou-se a inexistência, na literatura pesquisada, de instrumentos na língua portuguesa falada no Brasil que quantifiquem a exposição dos estudantes de Medicina a conteúdos e atividades correlatas às humanidades. Conhecer o perfil de exposição às humanidades dos estudantes de Medicina torna-se essencial, podendo subsidiar intervenções curriculares e/ou extracurriculares que incrementem os índices de exposição às atividades ligadas às humanidades. Este estudo teve por objetivo realizar a tradução, adaptação e validação do único instrumento estrangeiro que realiza esse tipo de mensuração7.

\section{MÉTODO}

Trata-se de estudo transversal de metodologia quantitativa, com proposta de adaptar transculturalmente e validar, do ponto de vista psicométrico, o Humanities Score ${ }^{7}$. Este estudo foi dividido em duas fases: realização da tradução e adaptação transcultural, e análise das propriedades psicométricas do instrumento traduzido. Ressalta-se que foi solicitada permissão ao autor principal para tradução do questionário. Para o estudo proposto, utilizou-se uma amostragem por conveniência, não probabilística, que considerou, para a validação semântica, o que preconizam Beaton et al. ${ }^{17}$ : a versão traduzida deve ser aplicada a uma amostra de 30 a 40 indivíduos da população-alvo. Para a avaliação psicométrica, atendeu-se à sugestão de Hair et al. ${ }^{18}$ que recomendam de cinco a dez indivíduos por item avaliado. Os critérios de inclusão foram: estar regularmente matriculado no primeiro, terceiro, quinto e sétimo períodos, compreender a proposta da pesquisa, aceitá-la e assinar o 
Termo de Consentimento Livre e Esclarecido (TCLE). Adotaramse os seguintes critérios de exclusão: não aceitação do TCLE, menoridade, recusa ou o preenchimento incompleto do questionário. Escolheram-se os períodos ímpares da fase préclínica do curso por se tratar de amostragem não probabilística por conveniência, incluindo os alunos mais acessíveis ao estudo por não estarem dispersos nos internatos hospitalares.

\section{$O$ instrumento}

Em revisão bibliográfica realizada nas bases de dados PubMed, Medline e SciELO, não foi encontrado um instrumento, em português, capaz de medir a exposição dos estudantes de Medicina às humanidades. Diante disso, optou-se por adaptar e validar o instrumento Humanities Score, elaborado por Mangione et al.7. A escolha baseou-se no fato de tratarse do único instrumento mencionado na literatura com essa abordagem.

\section{Tradução e adaptação transcultural}

O processo de tradução e adaptação transcultural foi baseado nos passos sugeridos por Beaton et al. ${ }^{17}$ em 2000 (Figura 1).

- A tradução do inglês para o português do Brasil foi realizada por dois tradutores juramentados, independentes entre si: um leigo no assunto abordado no estudo e o outro especializado em ensino de línguas estrangeiras, visando obter dois questionários traduzidos (T1 e T2).

- Compararam-se T1 e T2, e eventuais discrepâncias foram analisadas e discutidas pelos pesquisadores, originando a síntese da tradução em português $(\mathrm{T} 1+2)$.

- A síntese do questionário $(T 1+2)$ foi traduzida de volta para o inglês por um terceiro tradutor, produzindo a retrotradução (RT). Beaton et al. ${ }^{17}$ recomendam duas RTs, mas outros autores afirmam ser suficiente apenas uma ${ }^{19,20}$.

- A comparação do instrumento retrotraduzido ao instrumento original ocorreu mediante consenso entre os pesquisadores e um professor de língua inglesa, realizando ajustes para preservação do conteúdo do instrumento original (validação de conteúdo).

- Para obtenção da versão pré-final do questionário, realizou-se sua revisão, com especial atenção a elementos como instruções, acréscimo de itens, formato do instrumento, adequação da escala de respostas, existência de discrepâncias, entre outros (revisão pelos autores)

- O passo seguinte verificou a equivalência semântica e cultural entre a versão-fonte e a versão traduzida do questionário, pela aplicação do pré-teste (ou teste-piloto), no qual os respondentes escreveram o que compreenderam de cada item do instrumento, reescrevendo-o e/ou oferecendo sugestões de adequação. Essa etapa constituiu na adaptação semântica e transcultural que deu origem à versão final do instrumento traduzido (Figura 1).

\section{Validação psicométrica}

Para a avaliação das propriedades psicométricas do questionário traduzido, sua versão final, após o pré-teste, foi apresentada de forma autoaplicada a uma amostra por conveniência, composta de 258 estudantes do primeiro, terceiro, quinto e sétimo períodos de Medicina da Universidade José do Rosário Vellano (Unifenas-BH), entre fevereiro e abril de 2019. O questionário foi entregue aos alunos com o TCLE e demandou aproximadamente dez minutos para ser respondido de forma completa.

O instrumento finalizado continha 17 questões sociodemográficas, que abordavam idade, período em curso,

Figura 1. Fluxograma: tradução do Humanities Score.

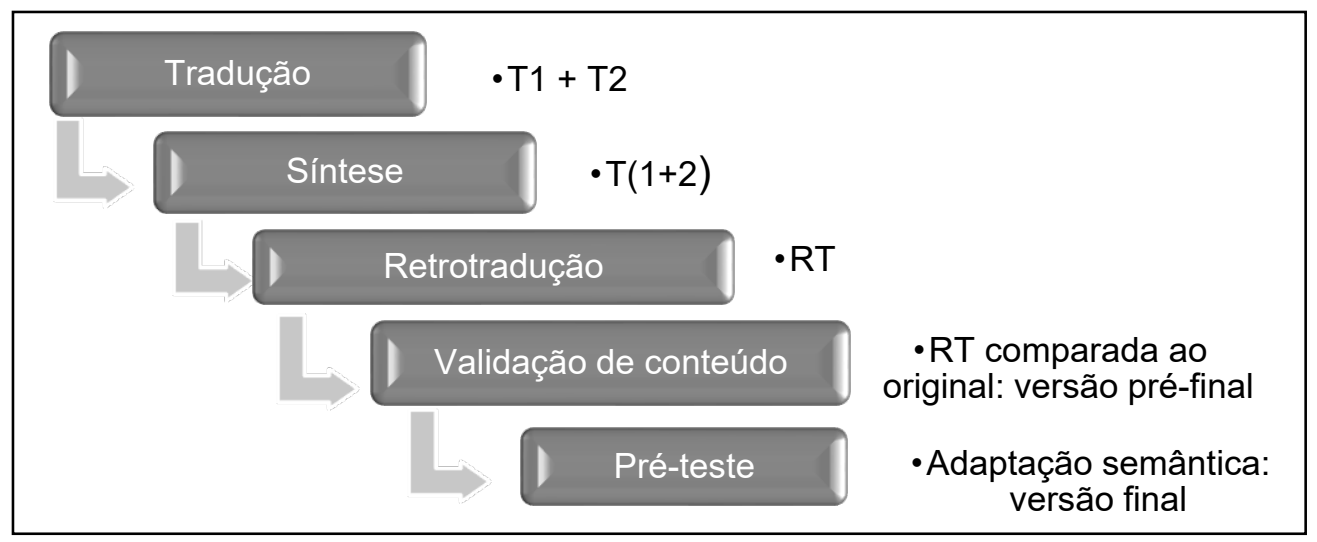

Fonte: Adaptada de Beaton et al. ${ }^{17}$. 
sexo, etnia, estado civil, local de nascimento, prática de religião, especialidade médica pretendida, realização prévia de outra graduação, conhecimento em língua estrangeira e com quem reside. Nele foram abordadas também realização de atividades extracurriculares, participação em trabalho social voluntário, prática de atividades físicas (essas atividades foram sugeridas para inclusão pelos autores do questionário original) e ainda atividades artísticas praticadas antes de iniciar o curso médico e sua eventual interrupção após o início do curso. Essa versão final continha também a versão traduzida e adaptada da Escala de Exposição às Humanidades $(\mathrm{EEH})$, constituída de 18 perguntas com respostas em escala de Likert com cinco opções (nunca, algumas vezes ao ano, mensalmente, semanalmente e diariamente) e cinco perguntas referentes à opinião sobre a inserção de humanidades no currículo médico, com respostas em escala de Likert com cinco opções (variando de discordo totalmente a concordo totalmente). Os itens com sentido inverso ao conjunto predominante das questões avaliadas tiveram suas pontuações invertidas. Para análise do índice de exposição, considerou-se a média total dos escores: quanto mais próxima de cinco, maior a exposição dos estudantes às humanidades.

A consistência interna do instrumento final foi determinada pelo cálculo do alfa de Cronbach, sendo considerados satisfatórios valores superiores a 0,6 $6^{21-23}$. Testaramse métodos de redução de complexidade do construto por meio de análise fatorial exploratória, mas foram desconsiderados por mostrarem-se insatisfatórios no caso desse instrumento.

\section{Aspectos éticos}

Este estudo foi aprovado pelo Comitê de Ética em Pesquisa (Coep) da Unifenas: Parecer $n^{\circ}$ 3.037.771. Todos os alunos envolvidos assinaram o TCLE.

\section{RESULTADOS}

\section{Tradução e adaptação cultural}

Após as etapas de tradução, síntese, retrotradução e revisão realizadas pelos autores, obteve-se a versão préfinal do instrumento, sem perda de conteúdo no transcurso dessas etapas. Visando à uniformização dos itens, foram implementadas alterações no formato das perguntas do questionário original: transformação das perguntas ramificadas em perguntas simples, sem modificação do seu teor e introdução da opção "nunca" nas respostas de frequência, que passaram a conter as opções nunca, algumas vezes ao ano, mensalmente, semanalmente e diariamente. Todas as escalas de Likert foram adaptadas para cinco opções de resposta ${ }^{24} \mathrm{e}$ pontuadas de 1 a 5 .
O pré-teste foi aplicado a 31 estudantes do oitavo período de medicina da Unifenas, selecionados aleatoriamente. Desses, $51,6 \%$ eram do sexo feminino, com média de idade de $24,9 \pm 5,4$ anos.

Essa etapa avaliou a compreensão dos estudantes em relação a cada item do instrumento. Foram apresentadas sugestões e/ou observações de modificação em quatro itens do questionário sociodemográfico: 1. no item"Antes de iniciar o curso de Medicina, participava de maneira regular de: atividades sociais voluntárias/grupos de estudos religiosos/atividades políticas/ meditação/outras", acrescentou-se a opção "nenhuma"; 2. no item "Antes de iniciar o curso de Medicina, frequentava espetáculos de canto, de dança, de teatro, shows ou concertos musicais, lia por prazer/nenhuma", acrescentou-se a opção "cinema"; 3. no item "Exerce atividades extracurriculares: nenhuma / estágio voluntário / estágio remunerado / empregado / autônomo", alteraram-se as alternativas de resposta para "sim" e "não", devido à possibilidade de o participante realizar outras atividades extracurriculares não contempladas nas opções; e 4. no item "Razão de interrupção da prática das atividades", acrescentou-se a opção"não se aplica”, para o caso de não terem sido interrompidas. $\mathrm{Na} \mathrm{EEH}$, o item "Frequência de comparecimento a concerto musical" foi modificado para "Frequência de comparecimento a eventos musicais". Na escala de opinião sobre a importância das humanidades, o item "Nem todos os currículos deveriam incluir ciências humanas" foi modificado para "Todos os currículos deveriam incluir ciências humanas".

\section{Avaliação das propriedades psicométricas do instrumento}

Para a avaliação das propriedades psicométricas da versão final do questionário traduzido, este foi distribuído para 258 estudantes de Medicina, recrutados no primeiro, terceiro, quinto e sétimo períodos do curso, entre fevereiro e abril de 2019, que concordaram em participar do estudo. Em virtude dos critérios de exclusão estabelecidos, sete estudantes foram excluídos por menoridade e outros 14 por terem respondido ao questionário de forma incompleta, permanecendo 237 participantes. Destes, 32,9\% eram do primeiro período, $24,1 \%$ do terceiro período, $22,8 \%$ do quinto período e $21,1 \%$ do sétimo período. A sociodemografia demonstrou um perfil constituído de $61,6 \%$ de mulheres, $74,7 \%$ de etnia branca, $95,8 \%$ de solteiros, $68,8 \%$ originários de Belo Horizonte ou cidades mineiras. Dos 237 alunos, 76,4\% declararam alguma prática religiosa, 16,5\% frequentaram outro curso superior antes do ingresso no curso de Medicina, 78,5\% tinham conhecimento de língua inglesa, $31,2 \%$ pretendiam fazer especialização em cirurgia e 18,6\% em clínica médica, 54,9\% exerciam atividades extracurriculares, e $65,8 \%$ praticavam atividades físicas individuais (Tabela 1). 
Tabela 1. Caracterização sociodemográfica dos participantes do estudo.

\begin{tabular}{|c|c|c|c|}
\hline \multicolumn{2}{|c|}{ Variáveis } & $\mathbf{n}$ & $\%$ \\
\hline \multirow{4}{*}{ Período em curso } & 10 Período & 78 & $32,9 \%$ \\
\hline & 3o Período & 57 & $24,1 \%$ \\
\hline & 70 Período & 51 & $22,8 \%$ \\
\hline & 50 Período & 50 & $21,1 \%$ \\
\hline \multirow{2}{*}{ Sexo } & Feminino & 146 & $61,6 \%$ \\
\hline & Masculino & 91 & $38,4 \%$ \\
\hline \multirow{5}{*}{ Etnia } & Branco & 174 & $73,4 \%$ \\
\hline & Pardo & 52 & $21,9 \%$ \\
\hline & Negro & 6 & $2,5 \%$ \\
\hline & Amarelo & 5 & $2,1 \%$ \\
\hline & Indígena & 0 & $0,0 \%$ \\
\hline \multirow{3}{*}{ Estado civil } & Solteiro & 227 & $95,8 \%$ \\
\hline & Casado & 6 & $2,5 \%$ \\
\hline & União estável & 4 & $1,7 \%$ \\
\hline \multirow{3}{*}{$\begin{array}{c}\text { Local de } \\
\text { nascimento }\end{array}$} & Interior de Minas Gerais & 81 & $34,2 \%$ \\
\hline & Belo Horizonte/RMBH & 72 & $30,4 \%$ \\
\hline & Outro estado & 82 & $34,6 \%$ \\
\hline \multirow{2}{*}{ Prática de religião } & Sim & 181 & $76,4 \%$ \\
\hline & Não & 56 & $23,6 \%$ \\
\hline \multirow{5}{*}{$\begin{array}{c}\text { Especialidade } \\
\text { médica } \\
\text { pretendida }\end{array}$} & Indeciso & 101 & $42,6 \%$ \\
\hline & Cirúrgica & 74 & $31,2 \%$ \\
\hline & Clínica & 44 & $18,6 \%$ \\
\hline & Saúde mental & 11 & $4,6 \%$ \\
\hline & Medicina da família & 6 & $2,5 \%$ \\
\hline \multirow{2}{*}{$\begin{array}{l}\text { Realizou outro } \\
\text { curso superior }\end{array}$} & Não & 198 & $83,5 \%$ \\
\hline & Sim & 39 & $16,5 \%$ \\
\hline \multirow{5}{*}{ Língua estrangeira } & Inglês & 186 & $78,5 \%$ \\
\hline & Espanhol & 55 & $23,2 \%$ \\
\hline & Outro idioma & 6 & $2,4 \%$ \\
\hline & Francês & 5 & $2,1 \%$ \\
\hline & Alemão & 2 & $0,8 \%$ \\
\hline \multirow{5}{*}{ Com quem reside } & $\begin{array}{c}\text { Colegas da faculdade de } \\
\text { Medicina }\end{array}$ & 89 & $37,6 \%$ \\
\hline & Familiares & 80 & $33,8 \%$ \\
\hline & Sozinho & 49 & $20,7 \%$ \\
\hline & Outros amigos & 17 & $7,2 \%$ \\
\hline & Cônjuge & 2 & $0,8 \%$ \\
\hline \multirow{2}{*}{$\begin{array}{l}\text { Exerce atividades } \\
\text { extracurriculares }\end{array}$} & Sim & 130 & $54,9 \%$ \\
\hline & Não & 104 & $43,9 \%$ \\
\hline \multirow{5}{*}{$\begin{array}{l}\text { Pratica atividades } \\
\text { físicas }\end{array}$} & Individuais & 131 & $55,3 \%$ \\
\hline & Coletivas & 88 & $37,1 \%$ \\
\hline & Nenhuma & 37 & $15,6 \%$ \\
\hline & Individuais com personal & 25 & $10,5 \%$ \\
\hline & Outras & 8 & $3,4 \%$ \\
\hline
\end{tabular}

$\mathrm{RMBH}=$ Região Metropolitana de Belo Horizonte.
A exposição às humanidades, antes de ingressarem na faculdade de Medicina, foi maior entre as atividades de exposição passiva (frequentar cinema e shows musicais e ler por prazer), e a eventual interrupção de alguma dessas atividades após o ingresso no curso de Medicina foi atribuída à existência de pouco tempo disponível, por 58,6\% dos respondentes (Gráfico 1).

Quando se empregaram metodologias de redução de complexidade do construto baseadas em análise fatorial exploratória, observou-se que essa análise não foi aplicável ao modelo, concluindo-se que o instrumento não poderia ser explicado pela metodologia de agrupamento em fatores, pois não foi possível estabelecer correlações satisfatórias entre itens. Adotou-se, portanto, o modelo unidimensional com os 17 itens de análise da exposição dos estudantes às humanidades, com as respostas em escala de Likert pontuadas de 1 a 5 . O cálculo do alfa de Cronbach resultou em um valor de 0,689 , considerado satisfatório (Tabela 2).

Quando se testou a validade do instrumento, foi possível observar que os estudantes apresentaram um índice médio de exposição às humanidades de 1,72 $\pm 0,37$, numa escala de exposição com valor máximo de 5 , variando de $24,8 \%$ a $68,6 \%$ de exposição. O hábito de estudar ouvindo música foi a atividade que obteve a maior média $(2,84)$, seguido por ler por prazer $(2,60)$, frequentar cultos religiosos $(2,27)$, dançar $(2,23)$ e frequentar eventos musicais $(2,15)$. As atividades relacionadas com produzir arte visual, visitar museus/galerias de arte, tocar algum instrumento musical, frequentar espetáculos de dança ou de teatro, escrever por prazer, meditar, participar de grupos de estudo religioso e realizar atividades sociais voluntárias e atividades políticas apresentaram índice de frequência com médias de escore variando de 1,25 a 1,75.

A exposição às humanidades foi influenciada pelo período do curso, com os estudantes do terceiro período apresentando escores significativamente mais elevados que os do sétimo período ( $p=0,011$ ). A exposição também foi influenciada pela realização de atividades voluntárias ( $p=$ $0,020)$, participação em grupos de estudos religiosos $(p=0,001)$, prática de meditação $(p=0,001)$ e participação em atividades políticas ( $p=0,001)$. As demais variáveis sociodemográficas (sexo, prática de religião, local de nascimento, especialidade pretendida, interrupção de atividades após o ingresso na faculdade e ter frequentado outro curso antes de iniciar Medicina) não influenciaram a exposição às humanidades. Em relação à opinião favorável dos estudantes sobre a importância atribuída às humanidades, as variáveis que tiveram uma influência positiva foram sexo feminino $(p=0,016)$, ter interrompido as atividades após iniciar o curso $(p=0,001)$ e participar de grupos de estudos religiosos $(p=0,016)$ (Tabela 3 ). 
Gráfico 1.Exposição dos alunos às humanidades antes do ingresso na faculdade.

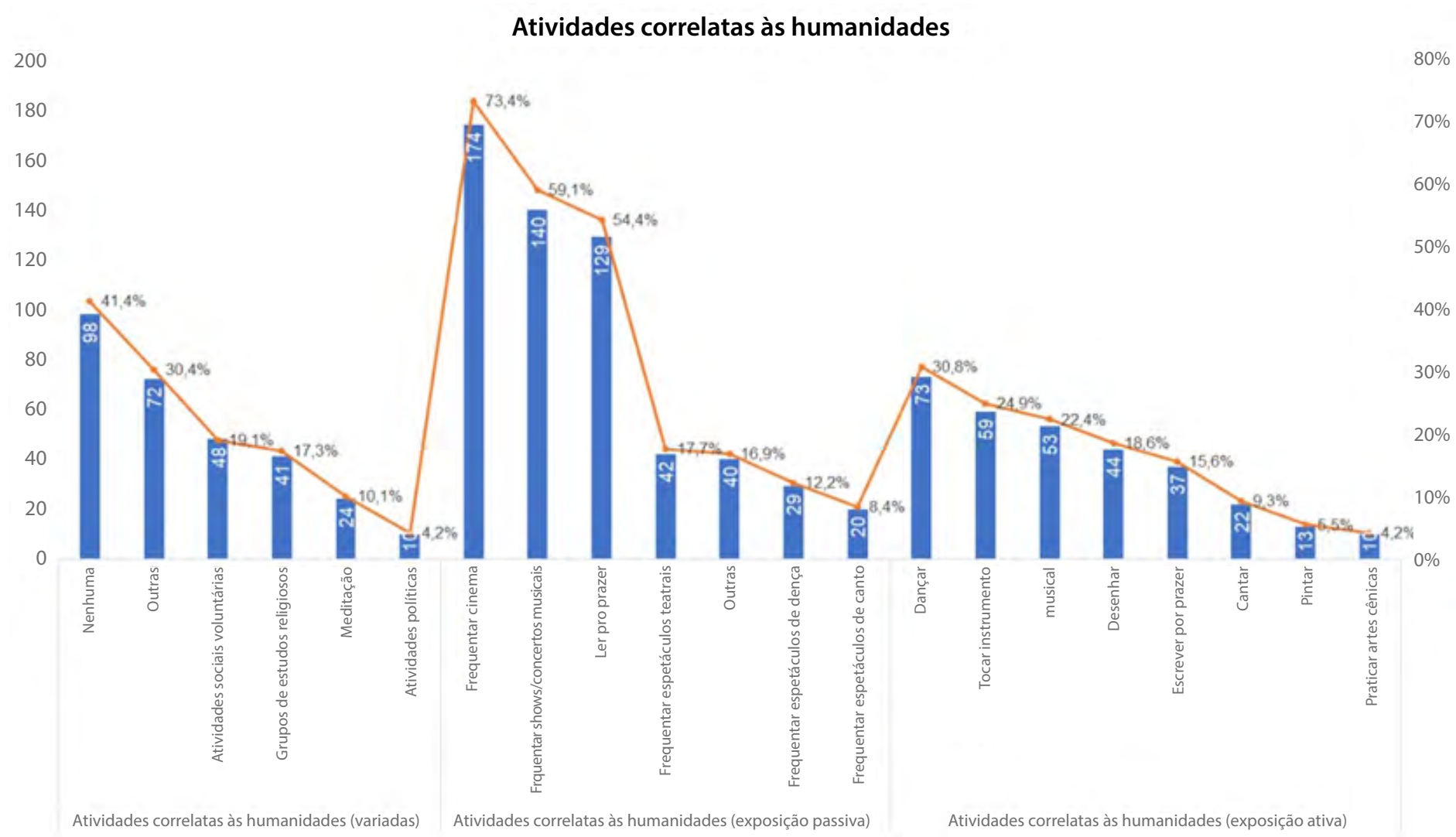

Tabela 2. Análise de confiabilidade da Escala de Exposição às Humanidades pelo teste alfa de Cronbach.

Item

Frequência de produção de arte visual (desenhar/esboçar/pintar/esculpir)

Frequência de visitação a museus de arte ou galerias de arte

Frequência de participação em coral ou grupo musical

Frequência de tocar algum instrumento musical

Frequência de visitação a espetáculos de dança

Frequência de estudar escutando música

Frequência de presença em eventos musicais

Frequência de dançar

Frequência de atuação em peças teatrais, exceto atividades escolares

Frequência em apresentações teatrais

Alfa de Cronbach se o item for removido

Frequência de escrever por prazer

Frequência de ler por prazer

Frequência em cultos religiosos

Frequência de prática de meditação

Frequência de participação em grupos de estudo de cunho religioso

Frequência de participação em atividades sociais voluntárias

Frequência de participação em atividades políticas
0,667

0,677

0,683

0,689

0,680

0,686

0,690

0,682

0,685

0,673

0,663

0,663

0,681

0,657

0,668

0,665

0,680

0,689


Tabela 3. Comparação entre a sociodemografia, o perfil de exposição às humanidades e o perfil de importância atribuída pelos alunos às humanidades.

\begin{tabular}{|c|c|c|c|c|c|c|c|}
\hline \multirow{2}{*}{\multicolumn{2}{|c|}{ Variáveis sociodemográficas }} & \multicolumn{3}{|c|}{ Escala de Exposição às Humanidades } & \multicolumn{3}{|c|}{ Escala de opinião } \\
\hline & & Média & $\begin{array}{l}\text { Desvio } \\
\text { padrãop }\end{array}$ & $\mathbf{p}$ & Média & $\begin{array}{l}\text { Desvio } \\
\text { padrão }\end{array}$ & $\mathbf{p}$ \\
\hline \multirow{4}{*}{ Período em curso } & 10 & 1,74 & 0,36 & \multirow{4}{*}{${ }^{*} 0,011$} & 4,23 & 0,72 & \multirow{4}{*}{0,108} \\
\hline & $3^{\circ}$ & 1,80 & 0,38 & & 4,03 & 0,80 & \\
\hline & $5^{\circ}$ & 1,64 & 0,33 & & 3,88 & 0,93 & \\
\hline & 70 & 1,62 & 0,35 & & 3,91 & 0,82 & \\
\hline \multirow{2}{*}{ Sexo } & Feminino & 1,74 & 0,35 & \multirow{2}{*}{0,073} & 4,19 & 0,65 & \multirow{2}{*}{0,016} \\
\hline & Masculino & 1,67 & 0,41 & & 3,82 & 0,99 & \\
\hline \multirow{2}{*}{ Pratica alguma religião } & Sim & 1,74 & 0,37 & \multirow{2}{*}{0,084} & 4,04 & 0,84 & \multirow{2}{*}{0,931} \\
\hline & Não & 1,64 & 0,36 & & 4,06 & 0,85 & \\
\hline \multirow{3}{*}{ Local de nascimento } & Belo Horizonte e RMBH & 1,71 & 0,38 & \multirow{3}{*}{0,705} & 4,08 & 0,77 & \multirow{3}{*}{0,424} \\
\hline & Interior de Minas Gerais & 1,68 & 0,32 & & 3,95 & 0,89 & \\
\hline & Outro estado & 1,75 & 0,42 & & 4,12 & 0,79 & \\
\hline \multirow{5}{*}{$\begin{array}{l}\text { Especialidade médica } \\
\text { pretendida }\end{array}$} & Clínica Médica & 1,66 & 0,26 & \multirow{5}{*}{0,051} & 4,00 & 0,92 & \multirow{5}{*}{0,081} \\
\hline & Cirúrgica & 1,75 & 0,41 & & 4,12 & 0,77 & \\
\hline & Saúde Mental & 1,81 & 0,35 & & 4,42 & 0,43 & \\
\hline & Medicina da família & 2,01 & 0,27 & & 4,60 & 0,42 & \\
\hline & Não decidi & 1,67 & 0,36 & & 3,94 & 0,83 & \\
\hline \multirow{2}{*}{ Interrompeu atividades } & Não & 1,64 & 0,36 & \multirow{2}{*}{0,061} & 3,74 & 0,91 & \multirow{2}{*}{0,001} \\
\hline & Sim & 1,74 & 0,38 & & 4,16 & 0,75 & \\
\hline \multirow{2}{*}{$\begin{array}{l}\text { Frequentou outro curso } \\
\text { superior }\end{array}$} & Sim & 1,82 & 0,48 & \multirow{2}{*}{0,311} & 4,21 & 0,80 & \multirow{2}{*}{0,115} \\
\hline & Não & 1,69 & 0,35 & & 4,02 & 0,82 & \\
\hline \multirow{2}{*}{$\begin{array}{l}\text { Realiza atividades sociais } \\
\text { voluntárias }\end{array}$} & Sim & 1,90 & 0,43 & \multirow{2}{*}{0,020} & 4,28 & 0,69 & \multirow{2}{*}{0,038} \\
\hline & Não & 1,67 & 0,35 & & 3,99 & 0,84 & \\
\hline \multirow{2}{*}{$\begin{array}{l}\text { Participa de grupos de } \\
\text { estudos religiosos }\end{array}$} & Sim & 2,02 & 0,36 & \multirow{2}{*}{0,001} & 4,34 & 0,72 & 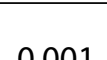 \\
\hline & Não & 1,65 & 0,34 & & 3,98 & 0,82 & 0,001 \\
\hline Pratica meditacão & Sim & 2,11 & 0,43 & 0.001 & 4,22 & 0,60 & 0473 \\
\hline PIdica meulaçau & Não & 1,67 & 0,34 & 0,001 & 4,03 & 0,84 & 0,423 \\
\hline Participa de atividades & Sim & 2,39 & 0,52 & 0.001 & 4,30 & 0,42 & 0524 \\
\hline políticas & Não & 1,68 & 0,34 & & 4,04 & 0,83 & $0,3<4$ \\
\hline Outras atividades & Sim & 1,79 & 0,37 & 0.013 & 4,09 & 0,73 & 0889 \\
\hline & Não & 1,68 & 0,37 & & 4,03 & 0,85 & \\
\hline
\end{tabular}

Testes estatísticos: variáveis com duas categorias: teste de Mann-Whitney; variáveis com três ou mais categorias: teste de Kruskall-Wallis. Nível de significância: $5 \%$.

$\mathrm{RMBH}=$ Região Metropolitana de Belo Horizonte.

\section{DISCUSSÃO}

O objetivo do presente estudo foi a tradução e adaptação sociocultural e validação psicométrica da $\mathrm{EEH}$. A originalidade desta pesquisa reside no fato de não se ter encontrado, na literatura brasileira, um instrumento que mensure essa exposição.

Trata-se de uma escala sucinta que aborda a exposição às várias formas de artes (literatura, teatro, música, dança, artes visuais, entre outras), permitindo obter uma ampla gama de informações relativas à exposição às humanidades ativa e passiva pelos estudantes de Medicina.

Segundo Borsa et al. $^{19}$, o processo de adaptação de instrumento deve considerar a clareza e pertinência dos conceitos pertencentes ao instrumento original, bem como a equivalência semântica, linguística e contextual dos itens. Nesse sentido, a EEH apresentou resultados satisfatórios após ter sido modificada e testada empiricamente em um grupo de estudantes de Medicina, demonstrando ser um instrumento de 
fácil entendimento e aplicação. Entretanto, apesar de os métodos qualitativos serem fundamentais para assegurar a adequação do processo de adaptação, faz-se necessário avaliar as propriedades psicométricas do instrumento ${ }^{20}$. Nesse estudo, a análise estatística conduzida demonstrou que a EEH apresentou distribuição de itens em uma única dimensão, sendo o valor de 0,689 encontrado para o alfa de Cronbach considerado satisfatório para o quesito confiabilidade. Considerou-se, para a análise da exposição, a média do total de escores obtidos nos itens com variação de 1 a 5: quanto mais próximo de 5 , maior a exposição.

No presente estudo, os resultados da aplicação da EEH demonstraram que a média de exposição dos estudantes foi de 1,72 (numa escala de 5). Resultados semelhantes foram obtidos por Mangione et al. ${ }^{7}$ em estudo multicêntrico, realizado em cinco escolas médicas dos Estados Unidos, que observaram média de escore de exposição às humanidades de 1,32 (em uma escala de 4).

Observou-se também que, apesar de a exposição às humanidades dos estudantes ser considerada baixa, eles julgam importante a presença das humanidades no currículo médico. Segundo Mairot et al. ${ }^{16}$, as humanidades fundamentadas em literatura, cinema, música, ópera, narrativas e outras modalidades, ao permitirem uma vivência construtiva de valores, experiências e diversidades, tornamse um instrumento de aprendizado e de crescimento pessoal. Dell Amore Filho et al. ${ }^{15}$, ao realizarem uma revisão sobre o ensino da humanização nas escolas médicas, observaram que existe uma expectativa de que o estudante de Medicina detenha características pessoais humanísticas e que consiga aplicá-las futuramente na prática profissional.

O debate sobre o papel fundamental das humanidades na capacidade de redimensionar saberes e discursos para o desenvolvimento de novos conceitos e novas formas de se fazer medicina está cada vez mais presente na atualidade ${ }^{25}$. Um médico humanista ou um "bom médico" deve ser capaz de aliar o conhecimento científico à capacidade interpretativa e à compreensão do outro, e possuir sensibilidade ética em sua prática clínica ${ }^{26}$. Nesse sentido, muito se tem estudado e também especulado sobre os efeitos potencialmente positivos que a exposição dos estudantes de Medicina a atividades e conteúdos relacionados às humanidades pode exercer sobre diversos aspectos da sua vida acadêmica e pessoal ${ }^{6,7}$. As várias formas de arte, como literatura, drama e música, refletem a alegria e a tristeza, sendo consideradas expressões da criatividade humana. Assim, a participação em alguma atividade artística, seja como espectador, leitor ou visualizador, faz parte da necessidade do indivíduo de tornar-se um ser humano completo ${ }^{26,27}$. Mangione et al. ${ }^{7}$ em 2018 observaram que a maior exposição às humanidades correlacionava-se a menores índices de burnout e a melhores índices de qualidades pessoais positivas (empatia, tolerância à ambiguidade, autoeficácia e inteligência emocional).

Infere-se, a partir desses resultados, que a exposição às humanidades proporciona melhora dos aspectos pessoais positivos e redução do burnout, podendo repercutir na melhora da qualidade de vida do estudante, o que, por sua vez, pode interferir de forma positiva na sua prática profissional. A relação construída com base em um profissional médico mais reflexivo e dotado de visão e posicionamento mais humanísticos reverterá na facilitação de maior adesão do paciente às condutas propostas e na possível obtenção de melhores índices de satisfação e até mesmo de melhores resultados clínicos.

Neste estudo, a observação de que o índice de exposição às humanidades diminui quando o aluno progride entre o primeiro e sétimo períodos é coerente com as informações fornecidas pelos alunos, ao alegarem interrupção de atividades correlatas às humanidades após o ingresso na faculdade por motivo de pouco tempo disponível. Esses dados são relevantes porque estudos pontuam que a "falta de tempo" é vista pelos estudantes de Medicina como responsável pela imposição de limitações para o lazer, para níveis desejáveis de descanso e para a prática de atividades extracurriculares, impactando a qualidade de vida deles ${ }^{28-30}$.

A prática das humanidades precisa ser estimulada ao longo do curso, satisfazendo a preocupação em formar profissionais médicos preparados tanto nos aspectos técnicocientíficos quanto no que concerne às relações humanas. Em revisão realizada por Mairot et al. $^{16}$, mencionam-se, como exemplos de atividades utilizáveis para essa finalidade, a observação detalhada de pinturas seguida de discussão e reflexão, com o intuito de desenvolver a capacidade de observação clínica do estudante; o uso da literatura para estimular a reflexão crítica dos discentes sobre valores humanísticos e a prática profissional; a utilização do teatro como ferramenta que facilita o aprendizado de habilidades de comunicação e narrativa de história e problemas clínicos comuns; e a utilização do cinema por meio do qual os alunos podem explorar seus valores, suas crenças e atitudes em relação às características do profissionalismo.

A importância da $\mathrm{EEH}$, que aborda vários aspectos relacionados às humanidades no curso de Medicina, reside na possibilidade de seu emprego em estudos futuros que forneçam elementos para o desenvolvimento de aspectos humanísticos do estudante e, por consequência, do profissional da medicina. Outrossim, possibilita a compreensão de fatores que interferem na exposição dos estudantes de Medicina às humanidades, favorecendo dessa forma o delineamento de estratégias curriculares mais adequadas ao perfil deles. 


\section{CONCLUSÕES}

Aversão final da EEH atendeu aos critérios de equivalência semântica, idiomática, cultural e conceitual, e apresentou adequada consistência interna, mostrando evidências de que o instrumento é adequado para avaliar a exposição dos estudantes de Medicina às humanidades. Como limitações do estudo, podemos citar que os participantes são de uma mesma instituição de ensino particular, pelo que sugerimos investigações adicionais com amostra mais diversificada. Como as evidências de validade não são definitivas, espera-se que novos estudos sejam realizados para confirmar os resultados deste estudo e que a EEH possa contribuir para o conhecimento do perfil de comportamento dos estudantes de Medicina em relação às humanidades e assim possibilitar o desenvolvimento de políticas de gestão e aprimoramento do currículo acadêmico.

\section{CONTRIBUIÇÃO DOS AUTORES}

Ilse Seubert Coelho Vieira participou do delineamento da pesquisa, da coleta de dados, da discussão dos resultados e da redação e revisão do artigo. Nathália Irffi Carvalho participou do delineamento da pesquisa, da coleta de dados e da discussão dos resultados. Antonio Carlos de Castro Toledo Júnior participou do delineamento da pesquisa e da análise dos dados. Eliane Perlatto Moura, além de orientar todo o processo referente à pesquisa, participou do delineamento da pesquisa, da coleta de dados, da discussão dos resultados e da redação e revisão do artigo.

\section{CONFLITO DE INTERESSES}

Declaramos não haver conflito de interesses.

\section{FINANCIAMENTO}

Declaramos não haver financiamento.

\section{REFERÊNCIAS}

1. Machado GDC. Arte y medicina: una relación de mutualismo facultativo. Mirabilia Medicinæ. 2018,10:1-11 [access in 23 sep 2019]. Available from: https://www.revistamirabilia.com/sites/default/files/medicinae/pdfs/ med2018-01-.

2. Azevedo VF. Uma breve história da arte na formação de médicos. látrico. 2015;15(35):40-8.

3. Chervel A, Compère MM. Les humanités dans I'histoire de l'enseignement français. Histoire de L'Éducation. 1997;74:5-38.

4. Craxì L, Giardina S, Spagnolo AG. A return to humane medicine: Osler's legacy. Infez Med. 2017;3:292-7.

5. Flexner A. Medical education in the United States and Canada. New York: Carnegie Foundation for the Advancement of Teaching; 1910.

6. Cooke M, Irby DM, Sullivan W, Ludmerer KM. American medical education 100 years after the Flexner report. NEJM. 2006;13:1339-44.
7. Mangione S, Chakraborti C, Staltari G, Harrison R, Tunkel AR, Liou KT, et al. Medical students' exposure to the humanities correlates with positive personal qualities and reduced burnout: a multi-institutional U. S. survey. J Gen Intern Med. 2018;33(5):628-34.

8. Catteral JS, Chapleau R, Iwanaga J. Involvement in the arts and human development: general involvement and intensive involvement in music and Theatre Arts. In: Fiske EB, editor. Champions of change: the impact of the arts on learning. Washington: Arts Education Partnership and President's Committee on the Arts and Humanities; 1999. p. 1-18 [access in 23 sep 2019]. Available from: http://artsedge.kennedy-center.org/ champions/pdfs/ChampsReport.pdf.

9. Lima RL, Soares MEC, Prado SN, Albuquerque GSC. Estresse do estudante de medicina e rendimento acadêmico. Rev Bras Educ Med. 2016;40(4):678-84.

10. Klier S. Lebensqualität von Medizinstudenten: Eine Studie zur Arbeitsbelastung, Gesundheit und der Bedeutung partnerschaftlicher Beziehungen im Medizinstudium [These]. Marburg: Universidade JustusLiebig Gießen; 2009.

11. Lyndon MP, Henning MA, Alyami H. Krishna S, Yu T-C, Hill AG. The impact of a revised curriculum on academic motivation, burnout, and quality of life among medical students. J Med Educ Curric Dev. 2017;4:1-8.

12. Song $P$, Jin $C$, Tang W. New medical education reform in China: towards healthy China 2030. Biosci Trends. 2017;11(4):366-9.

13. Cacchiarelli N, Catsicaris C, Cúneo MM, Damilano G, Dartiguelongue JB, Cunto $C$, et al. Las humanidades en la medicina: un camino para reflexionar acerca de la práctica clínica. Arch Argent Pediatr. 2017;115(5):99-104.

14. Ousager J, Johannessen $H$. Humanities in undergraduate medical education: a literature review. Acad Med. 2010;85(6):988-98.

15. Dell Amore Filho E, Dias RB, Toledo Jr ACC. Ações para a retomada do ensino da humanização nas escolas de Medicina. Rev Bras Educ Med. 2018;42(4):14-28.

16. Mairot LTS, Costa BBG, Heringer TPM, Borges RC, Moura, EP. As artes na educação médica: revisão sistemática da literatura. Rev Bras Educ Med. 2019;43(4):54-64

17. Beaton DE, Bombardier C, Guillemin F, Ferraz MB. Guidelines for the process of cross-cultural adaptation of self-report measures. Spine (Phila Pa 1976). 2000 Dec 15;25(24):3186-91.

18. Hair JF, Anderson RE, Tatham RL, Black WC. Análise multivariada de dados. 5 a ed. Porto Alegre: Bookman; 2005.

19. Borsa JC, Damásio BF, Bandeira DR. Adaptação e validação de instrumentos psicológicos entre culturas: algumas considerações. Paidéia. 2012;22(53):423-32

20. Eremenco SL, Cella D, Arnold BJ. A comprehensive method for the translation and cross-cultural validation of health status questionnaires. Eval Health Prof. 2005;28(2):212-32.

21. Fortes $C P D$, Araújo $A P Q C$. Check list para tradução e adaptação transcultura de questionários em saúde. Cad Saude Colet. 2019;27(2):202-9.

22. Landis JR, Koch GG. The measurement of observer agreement for categorical data. Biometrics. 1977;33(1):159-74.

23. Vieira S. Alfa de Cronbach. 2016 [access in 18 dec 2019]. Available from: http://soniavieira.blogspot.com/2015/10/alfa-de-cronbach.html.

24. Likert R. A technique for the measurement of attitudes. Arch Psychol 1932;22(140):5-55.

25. Rios, IC. Subjetividade contemporânea na educação médica: a formação humanística na medicina [tese]. São Paulo: Universidade de São Paulo; 2010.

26. Macnaughton J. The humanities in medical education: context, outcomes and structures. Med Humanit. 2000;26:23-30.

27. Mangione $S$, Kahn MJ. The old humanities and the new science at 100: Osler's enduring message. Cleve Clinic J Med. 2019;86(4):1-4.

28. Pasquali L. Psicometria: teoria dos testes na psicologia e na educação. 4a ed. Petrópolis: Vozes; 2011.

29. Figueiredo AM, Ribeiro GM, Reggianil ALM, Pinheiro BA, Leopoldo GO Duarte JAH, et al. Percepções dos estudantes de medicina da UFOP sobre sua qualidade de vida. Rev Bras Educ Med. 2014;38(4):435-43.

30. Chazan ACS, Campos MR. Qualidade de vida de estudantes de medicina medida pelo WHOQOL-bref - UERJ, 2010. Rev Bras Educ Med. 2013;37(3):376-84.

This is an Open Access article distributed under the terms of the Creative Commons Attribution License, which permits unrestricted use, distribution, and reproduction in any medium, provided the original work is properly cited. 